\title{
Exact controllability of linear neutral type systems by the moment problem approach
}

\author{
Rabah Rabah and Grigory M. Sklyar
}

\begin{abstract}
The problem of exact null-controllability is considered for a wide class of linear neutral type systems with distributed delay. The main tool of the analysis is the application of the moment problem approach and the theory of the basis property of exponential families. A complete characterization of this problem is given. The minimal time of controllability is specified. The results are based on the analysis of the Riesz basis property of eigenspaces of the neutral type systems in Hilbert space.
\end{abstract}

\section{INTRODUCTION}

Many applied problems from physics, mechanics, biology and other fields can be described by partial differential equations or delay differential equations. This leads to the construction and study of the infinite-dimensional system theory concerning also the systems with control. In this context the problem of controllability for distributed parameter systems leads to the study of the abstract controllability problem in infinite dimensional spaces, which may be formulated as follows in Hilbert spaces. Consider the abstract system

$$
\dot{x}=\mathcal{A} x+\mathcal{B} u,
$$

where $x(t) \in X, u(t) \in U, X$ and $U$ being Hilbert spaces, $\mathcal{A}$ is the generator of a $C_{0}$-semigroup $\mathrm{e}^{\mathcal{A} t}, \mathcal{B} \in \mathcal{L}(U, X)$ is a bounded operator. The problem of controllability is to find all the states $x_{T}$ that can be reached from a fixed initial state (say 0 ) at a finite the time $T$ by the choice of the controls $u(\cdot) \in L_{2}(0, T ; U)$. The mild solution of the system (1) is given by

$$
x(t)=\mathrm{e}^{\mathcal{A} t} x(0)+\int_{0}^{t} \mathrm{e}^{\mathcal{A}(t-\tau)} \mathcal{B} u(\tau) \mathrm{d} \tau .
$$

The reachability set from 0 at time $T$ is defined by

$$
\mathcal{R}_{T}=\left\{x: x=\int_{0}^{T} \mathrm{e}^{\mathcal{A} t} \mathcal{B} u(t) \mathrm{d} t, \quad u(\cdot) \in L_{2}(0, T ; U)\right\} .
$$

For finite dimensional systems the natural concept of controllability is when $\mathcal{R}_{T}=X$ (Kalman). For infinite dimensional systems, as has been pointed out by several authors (Fattorini, Triggiani, Russel, Balakrishnan and others) this concept is not realistic. It is easy to show that $\mathcal{R}_{T_{1}} \subset \mathcal{R}_{T_{2}}$ as $T_{1}<T_{2}$. In general, there is no universal time $T_{0}$ such that $\mathcal{R}_{T_{0}}=\mathcal{R}_{T}$

This work was supported by IRRCyN/École des Mines de Nantes. It is a short version of a paper publishied by SIAM [8].

R. Rabah is with IRCCyN, UMR CNRS 6597, École des Mines de Nantes, 4 rue Alfred Kastler, BP 2072244307 Nantes Cedex 3, France rabaheemn. fr

G. M. Sklyar is with the Institute of Mathematics, University of Szczecin, ulitsa Wielkopolska 15, 70451 Szczecin, Poland sklar@univ.szczecin.pl for all $T>T_{0}$. However, for several classes of systems important for application this property holds (hyperbolic type and neutral type systems). In these cases, a natural way to formulate the controllability problem is the following setting:

i) to find the maximal possible set $\mathcal{R}_{T}$ (depending on $T$ ),

ii) to find the minimal $T$ for which the set $\mathcal{R}_{T}$ becomes the maximal possible.

In order to obtain more profound and precise results by using this approach, it is important to consider a concrete class of systems and to use the specificity of this class. In this paper, we consider the problem of controllability for a general class of neutral systems with distributed delays given by the equation

$$
\left\{\begin{array}{l}
\frac{\mathrm{d}}{\mathrm{d} t}\left[z(t)-K z_{t}\right]=L z_{t}+B u(t), \quad t \geq 0 \\
z_{0}=f
\end{array}\right.
$$

where $z_{t}:[-1,0] \rightarrow \mathbb{C}^{n}$ is the history of $z$ defined by $z_{t}(s)=z(t+s)$. The difference and delay operators $K$ and $L$, respectively, are defined by $K f=A_{-1} f(-1)$ and

$$
L f=\int_{-1}^{0} A_{2}(\theta) \frac{\mathrm{d}}{\mathrm{d} \theta} f(\theta) \mathrm{d} \theta+\int_{-1}^{0} A_{3}(\theta) f(\theta) \mathrm{d} \theta
$$

for $f \in H^{1}\left([-1,0], \mathbb{C}^{n}\right)$, where $A_{-1}$ is a constant $n \times n$ matrix, $A_{2}, A_{3}$ are $n \times n$-matrices whose elements belong to $L_{2}(-1,0), B$ is a constant $n \times r$-matrix.

We consider the operator model of the neutral type system (2) introduced by Burns et al. in product spaces (see also [4]). The state space is $M_{2}\left(-1,0 ; \mathbb{C}^{n}\right)=\mathbb{C}^{n} \times L_{2}\left(-1,0 ; \mathbb{C}^{n}\right)$, shortly $M_{2}$, and (2) can be reformulated as

$$
\frac{\mathrm{d}}{\mathrm{d} t}\left(\begin{array}{c}
y(t) \\
z_{t}(\cdot)
\end{array}\right)=\mathcal{A}\left(\begin{array}{c}
y(t) \\
z_{t}(\cdot)
\end{array}\right)+\mathcal{B} u
$$

where

$$
\mathcal{A}\left(\begin{array}{c}
y(t) \\
z_{t}(\cdot)
\end{array}\right)=\left(\begin{array}{c}
L z_{t}(\cdot) \\
\mathrm{d} z_{t}(\theta) / \mathrm{d} \theta
\end{array}\right), \quad \mathcal{B} u(t)=\left(\begin{array}{c}
B u(t) \\
0
\end{array}\right) .
$$

The domain of $\mathcal{A}$ is $\mathcal{D}(\mathcal{A})$ with elements $x=\left(\begin{array}{c}y \\ z(\cdot)\end{array}\right)$ such that $z \in H^{1}\left([-1,0] ; \mathbb{C}^{n}\right)$ and $\left.y=z(0)-A_{-1} z(-1)\right\}$.

In the particular case when $A_{2}(\theta)=A_{3}(\theta)=0$, which corresponds to $L=0$, we will use the notation $\widetilde{\mathcal{A}}$ for $\mathcal{A}$.

Suppose that the initial condition for the system (2) is $z(t)=z_{0}(t), t \in[-1,0]$ and let us put $z_{t}(\theta)=z(t+\theta), \theta \in$ $[-1,0]$ and $y=z(0)-A_{-1} z(-1)$. The semigroup generated by $\mathcal{A}$ is given by

$$
\mathrm{e}^{\mathcal{A} t}\left(\begin{array}{c}
y \\
z_{0}(\cdot)
\end{array}\right)=\left(\begin{array}{c}
z_{t}(0)-A_{-1} z_{t}(-1) \\
z_{t}(\cdot)
\end{array}\right) .
$$


It can be shown that the reachability set $\mathcal{R}_{T}$ is such that $\mathcal{R}_{T} \subset \mathcal{D}(\mathcal{A})$ for all $T>0$. This is a consequence of the fact that for all $u(\cdot) \in L_{2}$ the corresponding solution of (2) is in $H^{1}$ and then the solution of (4) is in $\mathcal{D}(\mathcal{A})$ (see in [4] Proposition 2.2 for the existence of the solution and Corollary 2.7 for the property of the reachability subset). This naturally leads to the following definition of exact controllability.

Definition 1.1: The system (1) is exactly null-controllable by controls from $L_{2}$ at the time $T$ if $\mathcal{R}_{T}=\mathcal{D}(\mathcal{A})$. This means that the set of solutions of the system (2), $\{z(t), t \in[T-1, T]\}$ coincides with $H^{1}\left([T-1, T] ; \mathbb{C}^{n}\right)$.

This problem was the focus of attention of several authors in the 70s and 80s The main results were devoted to systems with one or several discrete delays. This may be explained by the fact that the explicit, in this case, form of solutions is known and, as a result, the semigroup describing the solutions of (2) is known explicitly.

The main result for the system:

$$
\dot{z}(t)-A_{-1} \dot{z}(t-h)=A_{0} z(t)+A_{1} z(t-h)+B u,
$$

is that the exact controllability holds if and only if

i) $\operatorname{rank}(\Delta(\lambda) \quad B)=n$,

ii) $\operatorname{rank}\left(\begin{array}{llll}B & A_{-1} B & \cdots & A_{-1}^{n-1} B\end{array}\right)=n$, where $\Delta(\lambda)=\lambda I-\lambda A_{-1} \mathrm{e}^{-\lambda h}-A_{0}-A_{1} \mathrm{e}^{-\lambda h}$. For the particular case of scalar control ( $B$ is $n \times 1$ matrix) the time of exact controllability is given in [5]: $T>n h$, where $h$ is the delay. For the general case, it is shown in [2] that the reachability set cannot increase for $T>n h$.

The case of non-commensurate delays with a distributed term was precisely studied in the paper by Yamamoto [15]. General conditions were given using the input-output technique. Conditions of approximate controllability (in [15], quasi-reachability) in the time domain were explicitly given for a system without distributed delay.

In contrast to the above mentioned works, we consider the model with distributed delays (2). In this case, we know only that the solution of (2) exists but the corresponding semigroup is not explicitly known. Then the technique using the explicit form of the solution, via an expression of the semigroup, cannot be used. So one needs another tool to analyze the controllability. In the similar situation of the controllability problems for hyperbolic systems, the powerful technique of the moment problem has been proved to be useful. It is caused by the fact that the operators corresponding to hyperbolic systems are as a rule skew-adjoint or close to skew-adjoint and then they possess a basis of eigenvectors. The expansion of the steering conditions in this basis allows the controllability problem for these systems to be reduced to a trigonometric problem with respect to some families of exponentials. Thus, the further analysis concerns the solvability of the non-Fourier trigonometric moment problem and is based on the profound theory of the Riesz bases of exponentials. This theory, originated by the famous PaleyWiener theorem, has essentially been developed in the last decades (see monographs by Avdonin and Ivanov [1], by Young [16], the paper [13] and references therein).
The main idea of our work is to apply the moment problem method to the analysis of controllability of neutral type systems. Note in this context that the case of neutral type systems differs essentially from those mentioned above since the operator $\mathcal{A}$ of the system is not skew-adjoint and moreover may not have a basis of eigenvectors or even generalized eigenvectors. The first element of our consideration is the spectral analysis of the operator model (4) given in our previous works, together with A.V. Rezounenko [9], [11]. In these papers, it is shown that, under the condition that the matrix $A_{-1}$ is not singular, the operator $\mathcal{A}$ (even if it does not verify the Riesz basis property) possesses a Riesz basis of finite-dimensional invariant subspaces. This allows the construction of a special Riesz basis in the space $M_{2}$ in which the steering conditions:

$$
\left(\begin{array}{c}
y \\
z(\cdot)
\end{array}\right)=\int_{0}^{T} \mathrm{e}^{\mathcal{A}(T-\tau)} \mathcal{B} u(\tau) \mathrm{d} \tau
$$

take the form of a moment problem quadratically close to some special non-Fourier moment problems with respect to a family of quasi-polynomials. These questions are considered in Section II and Section III. Let us notice that the detailed attention accorded to the construction of the needed Riesz basis is essentially motivated by the fact that, in the general case, the operator $\mathcal{A}$ may not possess a spectral Riesz basis. Otherwise, for example if the eigenvalues of matrix $A_{-1}$ are simple, our construction would be much simpler. The main tool of the analysis of solvability of the obtained moment problem is based on the theory of families of exponentials [1], [16]. The basic elements of this approach used in our paper are given in Section IV. Below we give a complete analysis of the controllability problem for neutral type systems. In the course of the main part of the work, we consider the case when $\operatorname{det} A_{-1} \neq 0$. In this case, the controllability of system (2) is equivalent to the solvability of the moment problem obtained in Section II. We consider first the single input case in Section V and give not only the conditions of exact null-controllability but also determine the time of controllability. These results are an extension of the result obtained in [5]. In Section VI, we consider the solvability of the moment problem for the multivariable case $(\operatorname{dim} B=n \times r, r>1)$. We introduce some special indices $m_{1}$ and $\bar{m}$ which enable the moment problem to be characterized. We show that the exact null-controllability holds for time $T>\bar{m}$ and does not hold for $T<m_{1}$. Finally, we complete the results on controllability by getting rid of the assumption $\operatorname{det} A_{-1} \neq 0$ in Section VII. We then obtain the precise time of controllability using the first controllability index of the par $\left(A_{-1}, B\right)$, say $n_{1}$ (cf. for example [14, Chapter 5]).

This a short version of the paper [8]. For details and for the proofs, we refer to the original paper.

\section{THE MOMENT PROBLEM}

The main tool is to consider the null-controllability problem as a problem of moments. In order to formulate the moment problem we need a Riesz basis in the Hilbert 
space $M_{2}$. We recall that a Riesz basis is a basis which may be transformed to an orthogonal basis with respect to another equivalent scalar product. Each Riesz basis possesses a biorthogonal basis. Let $\{\varphi\}$ be a Riesz basis in $M_{2}$ and $\{\psi\}$ the corresponding biorthogonal basis. Then for each $x \in M_{2}$ we have $x=\sum_{\varphi \in\{\varphi\}}\langle x, \psi\rangle \varphi$.

A state $x=\left(\begin{array}{c}y \\ z(\cdot)\end{array}\right) \in M_{2}$ is reachable at time $T$ by a control $u(\cdot) \in L_{2}\left(0, T ; \mathbb{C}^{r}\right)$ if and only if the steering condition (5) holds. This steering condition may be expanded using the basis $\{\varphi\}$. A state $x$ is reachable if and only if

$$
\sum_{\varphi \in\{\varphi\}}\langle x, \psi\rangle \varphi=\sum_{\varphi \in\{\varphi\}} \int_{0}^{T}\left\langle\mathrm{e}^{\mathcal{A} t} \mathcal{B} u(t), \psi\right\rangle \mathrm{d} t \varphi,
$$

for some $u(\cdot) \in L_{2}\left(-h, 0 ; \mathbb{R}^{r}\right)$. Then the steering condition (5) can be substituted by the following system of equalities

$$
\langle x, \psi\rangle=\int_{0}^{T}\left\langle\mathrm{e}^{\mathcal{A} t} \mathcal{B} u(t), \psi\right\rangle \mathrm{d} t, \quad \psi \in\{\psi\} .
$$

Let $\left\{b_{1}, \ldots, b_{r}\right\}$ be an arbitrary basis in $\operatorname{Im} B$, the image of the matrix $B$ and $\mathbf{b}_{i}=\left(\begin{array}{c}b_{i} \\ 0\end{array}\right) \in M_{2}, i=1, \ldots, r$. Then the right hand side of (6) takes the form

$$
\int_{0}^{T}\left\langle\mathrm{e}^{\mathcal{A} t} \mathcal{B} u(t), \psi\right\rangle \mathrm{d} t=\sum_{i=1}^{r} \int_{0}^{T}\left\langle\mathrm{e}^{\mathcal{A} t} \mathbf{b}_{i}, \psi\right\rangle u_{i}(t) \mathrm{d} t .
$$

Effectiveness of the proposed approach becomes obvious if we assume that the operator $\mathcal{A}$ possess a Riesz basis of eigenvector. This situation is characteristic, for example, for control systems of hyperbolic type when $\mathcal{A}$ is skewadjoint $\left(\mathcal{A}^{*}=-\mathcal{A}\right)$ and has a compact resolvent (see, for example, [1], [16], [17]). Let in this case $\left\{\varphi_{k}\right\}, k \in \mathbb{N}$, be a orthonormal eigenbasis with $\mathcal{A} \varphi_{k}=i \lambda_{k} \varphi_{k}, \lambda_{k} \in \mathbb{R}$. Assuming for simplicity $r=1, b_{1}=b=\sum_{k} \alpha_{k} \varphi_{k}, \alpha_{k} \neq 0$, we have from (6)-(7)

$$
\frac{x_{k}}{\alpha_{k}}=\int_{0}^{T} e^{-i \lambda_{k} t} u(t) d t, \quad k \in \mathbb{N}
$$

where $x=\sum_{k} x_{k} \varphi_{k}$. Equalities (6) are a non-Fourier trigonometric moment problem whose solvability is closely connected with the property for the family of exponentials $e^{-i \lambda_{k} t}, k \in \mathbb{N}$, to form a Riesz basis on the interval $[0, T]$ ([1]). In particular, if $e^{-i \lambda_{k} t}$ forms a Riesz basis of $L_{2}\left[0, T_{0}\right]$ then one has

$$
\mathcal{R}_{T}=\left\{x: \sum_{k}\left(\frac{x_{k}}{\alpha_{k}}\right)^{2}<\infty\right\} \quad \text { for all } T \geq T_{0} .
$$

Obviously formula (7) gives the complete answer to the both items of the controllability problem.

The situation is more complicated if we have a basis of generalized eigenvectors. In this case instead of exponential, we have quasi-polynomials. In this case the Riesz basis property in $L_{2}$ is more complex. Returning now to neutral type systems we observe that the operator $\mathcal{A}$ given in (2) is not skew-adjoint and, moreover, does not possess a basis even of generalized eigenvectors. So the choice of a proper
Riesz basis in context of formulas (6)-(7) is an essentially more complicated problem.

\section{THE CHOICE OF BASIS}

In order to design the needed basis for our case we use the spectral properties of the operator $\mathcal{A}$ obtained in [11]. Let $\mu_{1}, \ldots, \mu_{\ell}, \quad \mu_{i} \neq \mu_{j}$ be eigenvalues of $A_{-1}$ and let the integers $p_{m}$ be defined as $: \operatorname{dim}\left(A_{-1}-\mu_{m} I\right)^{n}=p_{m}, m=$ $1, \ldots, \ell$. Denote by

$\lambda_{m}^{(k)}=\left(\ln \left|\mu_{m}\right|+\mathrm{i}\left(\arg \mu_{m}+2 \pi k\right)\right) ; m=1, \ldots, \ell ; k \in \mathbb{Z}$,

and let $L_{m}^{(k)}$ be the circles of the fixed radius $r \leq r_{0}=$ $\frac{1}{3} \min \left|\lambda_{m}^{(k)}-\lambda_{i}^{(j)}\right|$ centered at $\lambda_{m}^{(k)}$.

Let $\left\{V_{m}^{(k)}\right\}_{\substack{k \in \mathbb{Z} \\ m=1, \ldots, \ell}}$ be a family of $\mathcal{A}$-invariant subspaces given by

$$
V_{m}^{(k)}=P_{m}^{(k)} M_{2}, \quad P_{m}^{(k)}=\frac{1}{2 \pi \mathrm{i}} \int_{L_{m}^{(k)}} R(\mathcal{A}, \lambda) d \lambda .
$$

The following theorem plays an essential role in our approach

Theorem 3.1: [9] There exists $N_{0}$ large enough such that for any $N \geq N_{0}$

i) $\operatorname{dim} V_{m}^{(k)}=p_{m}, k \geq N$,

ii) the family $\left\{V_{m}^{(k)}\right\}_{\substack{|k| \geq N \\ m=1, \ldots, \ell}} \cup \widehat{V}_{N}$ forms a Riesz basis (of subspaces) in $M_{2}$, where $\widehat{V}_{N}$ is a finite-dimensional subspace $\left(\operatorname{dim} \widehat{V}_{N}=2(N+1) n\right)$ spanned by all generalized eigenvectors corresponding to all eigenvalues of $\mathcal{A}$ located outside of all circles $L_{m}^{(k)},|k| \geq N, m=1, \ldots, \ell$.

Using this theorem we construct a Riesz basis $\{\varphi\}$ which elements can be represented by

$$
\{\varphi\}=\left\{\varphi_{m, k}^{j, s},|k|>N\right\} \cup\left\{\widehat{\varphi}_{m}^{j, s}\right\} .
$$

The corresponding biorthogonal basis is $\{\psi\}$ with the same indices. More precisely, there exists $N_{1}$ such that for any given $N>N_{1}$ and $m=1, \ldots, \ell$ the collection $\left\{\varphi_{m, k}^{j, s}, j=\right.$ $\left.1, \ldots, \nu_{m} ; s=1, \ldots, p_{m, j}\right\}$ is a special basis of $V_{m}^{(k)}$. It is important to notice that in the construction of the basis, one uses the fact that the operator $\widetilde{\mathcal{A}}$ has a Riesz basis of generalized eigenvectors. In this basis equalities (6)(7) turns into a moment problem with respect to a special collection of quasi-polynomials. Analyzing the mentioned moment problem by means of the methods given in [1] we obtain our main results concerning the null-controllability problem. The chosen basis $\{\varphi\}$ and the biorthogonal $\{\psi\}$ will be used in our further analysis of the steering conditions by the moment problem method. We distinguish the infinite part of the basis $\left\{\psi_{m, k}^{j, s},|k|>N\right\}$ and the finite part $\left\{\widehat{\varphi}_{m}^{j, s}\right\}$.

In this context, we notice that the construction of a proper basis becomes rather complicated only in the case when the spectrum of matrix $A_{-1}$ is not simple and, as a consequence, the operator $\mathcal{A}$ may not possess a spectral Riesz basis. If all eigenvalues of $A_{-1}$ are simple, the basis $\{\varphi\}$ constructed in this Section coincides with a spectral basis of $\mathcal{A}$. 


\section{THE MOMENT PROBLEM AND THE RIESZ BASIS} PROPERTY OF A FAMILY OF QUASI-POLYNOMIALS

In order to use the results of Section II, we assume that the matrix $A_{-1}$ is not singular.

Let us expand the steering condition (5) with respect to the basis $\{\varphi\}$ and to the biorthogonal basis $\{\psi\}$. Consider a state $x=\left(\begin{array}{c}y \\ z(\cdot)\end{array}\right) \in M_{2}$, this state is reachable at time $T$ if and only if

$$
\sum_{\varphi \in\{\varphi\}}\langle x, \psi\rangle \varphi=\sum_{\varphi \in\{\varphi\}} \int_{0}^{T}\left\langle\mathrm{e}^{\mathcal{A} t} \mathcal{B} u(t), \psi\right\rangle \mathrm{d} t \varphi,
$$

where $u(\cdot) \in L_{2}\left(-1,0 ; \mathbb{C}^{r}\right)$. Then the steering condition (5) can be substituted by the following system of equalities (6)-(7). The infinite part of the system (6) corresponding to $\psi \in\left\{\psi_{m, k}^{j, s}\right\},|k|>N$ reads as

$$
\begin{aligned}
& k\left\langle\left(\begin{array}{c}
y_{T} \\
z_{T}(\cdot)
\end{array}\right), \psi_{m, k}^{j, s}\right\rangle \\
& =\sum_{d=1}^{r} \int_{0}^{T}\left(\mathrm{e}^{\lambda_{m}^{(k)} t} q_{m, k}^{j, s, d}(t)+f_{m, k}^{j, s, d}(t)\right) u_{d}(t) \mathrm{d} t
\end{aligned}
$$

Moreover, one can show that, the functions $\{q(t)\}$ and $\{q(t)\}$ verify the following properties:

P1) The coefficients of the polynomials $\{q(t)\}$ are uniformly bounded as $|k|>N$;

P2) The set of leading coefficients of the nontrivial polynomials $\{q(t)\}$ does not have a limit point at 0 ;

P3) $\sum_{|k|>N}\left|f_{m, k}^{j, s, d}(t)\right|^{2}<\alpha<\infty, t \in[0, T], \alpha>0$.

Therefore, the finite part of the system (6) corresponding to $\psi \in\left\{\widehat{\psi}_{m}^{j, s}\right\}$ reads as

$$
\left\langle\left(\begin{array}{c}
y_{T} \\
z_{T}(\cdot)
\end{array}\right), \widehat{\psi}_{m}^{j, s}\right\rangle=\sum_{d=1}^{r} \int_{0}^{T} \mathrm{e}^{\widehat{\lambda}_{m} t} \widehat{q}_{m, k}^{j, s, d}(t) u_{d}(t) \mathrm{d} t .
$$

Thus, we observe that the state $\left(\begin{array}{c}y \\ z(\cdot)\end{array}\right) \in M_{2}$ is reachable from 0 at the time $T>0$ if and only if the equalities (10) and (11) hold for some controls $u_{d}(\cdot) \in L_{2}(0, T), d=1, \ldots, r$. These equalities pose a kind of moment problem, namely a vector moment problem, which is the main object of our further analysis.

Our analysis is based on the theory of families of exponential developed by Avdonin and Ivanov in [1]. We are particularly interested in the basis properties of such families.

Let $\delta_{1}, \ldots, \delta_{\ell}$ be different, modulus $2 \pi \mathrm{i}$, complex numbers, $m_{1}, \ldots, m_{\ell}$ and $N$ be natural integers. Let us denote by $\widetilde{\mathcal{E}}_{N}$ the family

$$
\left\{\mathrm{e}^{\left(\delta_{s}+2 \pi \mathrm{i} k\right) t}, t \mathrm{e}^{\left(\delta_{s}+2 \pi \mathrm{i} k\right) t}, \ldots, t^{m_{s}-1} \mathrm{e}^{\left(\delta_{s}+2 \pi \mathrm{i} k\right) t}\right\}_{\substack{|k|>N \\ s=1, \ldots, \ell}}
$$

Next, let $\varepsilon_{1}, \ldots, \varepsilon_{r}$ be another collection of different complex numbers such that $\varepsilon_{j} \neq \delta_{s}+2 \pi \mathrm{i} k, j=1, \ldots, r ; s=$ $1, \ldots, \ell ;|k|>N$ and $m_{1}^{\prime}, \ldots, m_{r}^{\prime}$ be positive integers. Let us denote by $\mathcal{E}_{0}$ the collection

$$
\mathcal{E}_{0}=\left\{\mathrm{e}^{\varepsilon_{j} t}, t \mathrm{e}^{\varepsilon_{j} t}, \ldots, t^{m_{s}^{\prime}-1} \mathrm{e}^{\varepsilon_{j} t}\right\}_{j=1, \ldots, r} .
$$

The following theorem is the main tool of our further analysis.

Theorem 4.1: i) If $\sum_{j=1}^{r} m_{j}^{\prime}=(2 N+1) \sum_{s=1}^{\ell} m_{s}$ then the family $\mathcal{E}=\widetilde{\mathcal{E}}_{N} \cup \mathcal{E}_{0}$ constitutes a Riesz basis in $L_{2}\left(0, \sum_{s=1}^{\ell} m_{s}\right)$ ii) If $T>\sum_{s=1}^{\ell} m_{s}$ then, independently of the number of elements in $\mathcal{E}_{0}$, the family $\mathcal{E}$ forms a Riesz basis of the closure of its linear span in the space $L_{2}(0, T)$.

Now we apply Theorem 4.1 to the collection of functions appearing in (10). Let us fix $d \in\{1, \ldots, r\}$ and choose an arbitrary subset $L \subset\{1, \ldots, \ell\}$. Next, for any $m \in$ $L$ we choose $j(m) \in\left\{1, \ldots, \nu_{m}\right\}$ and denote $J(L)=$ $\{j(m)\}_{m \in L}$. Finally, for any couple $(m, j(m)), m \in L$, we put $\pi_{m, j(m)}=\operatorname{deg} q_{m, k}^{j(m), 1, d}(t)+1$.

Let us precise that this degree does not depend on $k$.

Theorem 4.2: For any choice of $d, L, J(L)$, for any $p_{m, j(m)}^{\prime}$, such that $1 \leq p_{m, j(m)}^{\prime} \leq \pi_{m, j(m)}$, and for any $T \geq n^{\prime}=\sum_{m \in L} p_{m, j(m)}^{\prime}$ the collection of functions

$$
\Phi_{1}=\left\{\mathrm{e}^{\lambda_{m}^{(k)} t} q_{m, k}^{j(m), s, d}(t)\right\}_{|k|>N ; m \in L ; s \in S}
$$

where $S=\pi_{m, j(m)}-p_{m, j(m)}^{\prime}+1, \ldots, \pi_{m, j(m)}$, constitutes a Riesz basis of $\mathrm{Cl} \operatorname{Lin} \Phi_{1}$ in $L_{2}(0, T)$.

If in addition $N$ is large enough, then the family

$$
\Phi_{2}=\left\{\mathrm{e}^{\lambda_{m}^{(k)} t} q_{m, k}^{j(m), s, d}(t)+f_{m, k}^{j(m), s, d}(t)\right\}_{|k|>N ; m \in L ; s \in S}
$$

also forms a Riesz basis of $\mathrm{Cl} \operatorname{Lin} \Phi_{2}$ in $L_{2}(0, T)$.

If $T=n^{\prime}$ the subspaces $\mathrm{Cl} \operatorname{Lin} \Phi_{1}$ and $\operatorname{Cl} \operatorname{Lin} \Phi_{2}$ are of finite codimension $(2 N+1) n^{\prime}$ in $L_{2}\left(0, n^{\prime}\right)$.

\section{THE SINGLE CONTROL CASE}

Let us study the solvability of the systems of equalities (10) and (11). We assume again that the matrix $A_{-1}$ is not singular, det $A_{-1} \neq 0$.

Consider the sequence

$$
\left\{\int_{0}^{T}\left(\mathrm{e}^{\lambda_{m}^{(k)} t} q_{m, k}^{j(m), s, d}(t)+f_{m, k}^{j(m), s, d}(t)\right) u(t) \mathrm{d} t\right\}
$$

for $|k|>N, s=1, \ldots, p_{m, j(m)}$ and any fixed $d, m, j$ and $u(\cdot) \in L_{2}(0, T)$. It follows from Theorem 4.2 that all nonzero functions of the collection

$$
\left\{\mathrm{e}^{\lambda_{m}^{(k)} t} q_{m, k}^{j(m), s, d}(t),|k|>N ; s=1, \ldots, p_{m, j}\right\}
$$

form a Riesz basis of their linear span in $L_{2}\left(0, T^{\prime}\right)$ if $T^{\prime}$ is large enough. Therefore the first term of (12) belongs to the class $\ell_{2}$. On the other hand, the second term also belongs to $\ell_{2}$. This gives the following Proposition.

Proposition 5.1: If the state $\left(\begin{array}{c}y_{T} \\ z_{T}(\cdot)\end{array}\right)$ is reachable from 0 by the system (4) then it satisfies the following equivalent conditions

C1) $\sum_{\substack{|k|>N \\ m, j, s}} k^{2}\left|\left\langle\left(\begin{array}{c}y_{T} \\ z_{T}(\cdot)\end{array}\right), \psi_{m, k}^{j, s}\right\rangle\right|^{2}<\infty$, 


$$
\begin{aligned}
& \text { C2) } \sum_{\substack{|k|>N \\
m=1, \ldots, \ell}} k^{2}\left\|P_{m}^{(k)}\left(\begin{array}{c}
y_{T} \\
z_{T}(\cdot)
\end{array}\right)\right\|^{2}<\infty, \\
& \text { C3) }\left(\begin{array}{c}
y_{T} \\
z_{T}(\cdot)
\end{array}\right) \in \mathcal{D}(\mathcal{A}) .
\end{aligned}
$$

From Proposition 5.1 it follows once more, as was pointed out in the Introduction (see also [4]), that the set $\mathcal{R}_{T}$ of the states reachable from 0 by virtue of the system (4) and controls from $L_{2}(0, T)$ is always a subset of $\mathcal{D}(\mathcal{A})$. This justifies also the Definition 1.1 given in the Introduction: the system (4) is said to be null-controllable at the time $T$ if $\mathcal{R}_{T}=\mathcal{D}(\mathcal{A})$. Next, we give the necessary conditions of null-controllability.

Theorem 5.2: Assume that the system (4) is nullcontrollable by controls from $L_{2}(0, T)$ for some $T>0$. Then the following two conditions hold:

i) There is no $\lambda \in \mathbb{C}$ and $y \in \mathbb{C}^{n}, y \neq 0$ such that $\Delta_{\mathcal{A}}^{*}(\lambda) y=0$ and $B^{*} y=0$, or equivalently $\operatorname{rank}\left(\Delta_{\mathcal{A}}(\lambda) \quad B\right)=n$ for all $\lambda \in \mathbb{C}$. Here

$$
\Delta_{\mathcal{A}}(\lambda)=\lambda I-\lambda \mathrm{e}^{-\lambda} A_{-1}-L(s),
$$

where the matrix $L(s)$ is obtained from the operator $L$ of the system (2) given by (3), namely

$$
L(s)=\lambda \int_{-1}^{0} \mathrm{e}^{\lambda s} A_{2}(s) \mathrm{d} s+\int_{-1}^{0} \mathrm{e}^{\lambda s} A_{3}(s) \mathrm{d} s .
$$

ii) There is no $\mu \in \sigma\left(A_{-1}\right)$ and $y \in \mathbb{C}^{n}, y \neq 0$ such that $A_{-1}^{*} y=\bar{\mu} y$ and $B^{*} y=0$, or equivalently $\operatorname{rank}\left(\begin{array}{lll}B & A_{-1} B & \cdots, A_{-1}^{n-1} B\end{array}\right)=n$.

Now we are ready to give the first important result of our work.

Theorem 5.3: Let the conditions i) and ii) of Theorem 5.2 hold. Then

i) The system (4) is null-controllable at the time $T$ as $T>n$;

ii) If the system (4) is of single control $(r=1)$, then the estimation of the time of controllability in i) is exact, i.e. the system is not controllable at time $T=n$.

If the delay is $h$ instead of 1 , the time of exact controllability is $T=n h$.

\section{Controllability IN THE MUltivariable CASE}

Let us now consider the multivariable case: $\operatorname{dim} B=r$ with also the assumption that the matrix $A_{-1}$ is not singular, $\operatorname{det} A_{-1} \neq 0$.

Let $\left\{b_{1}, \ldots, b_{r}\right\}$ be an arbitrary basis noted $\beta$. Let us introduce a set of integers. We denote by $B_{i}=$ $\left(b_{i+1}, \ldots, b_{r}\right), i=0,1, \ldots, r-1$, which gives in particular $B_{0}=B$ and $B_{r-1}=\left(b_{r}\right)$ and we put formally $B_{r}=0$. Let us put

$$
\mathcal{C}_{i}=\left(\begin{array}{llll}
B_{i} & A_{-1} B_{i} & \cdots & A_{-1}^{n-1} B_{i}
\end{array}\right) i=0,1, \ldots, r-1
$$

We need in the sequel the integers

$$
m_{i}^{\beta}=\operatorname{rank} \mathcal{C}_{i-1}-\operatorname{rank} \mathcal{C}_{i},
$$

corresponding to the basis $\beta$. Let us denote by

$$
m_{1}=\max _{\beta} m_{1}^{\beta}, \quad \bar{m}=\min _{\beta} \max _{i} m_{i}^{\beta},
$$

for all possible choices of a basis $\beta$. It is easy to show that for all $\beta$, there exists $i$ such that $m_{i}^{\beta} \geq$ $m_{1}$ and then $\bar{m} \geq m_{1}$. Indeed, assume that $m_{1}$ is realized on the basis $\beta=\left\{b_{1}, \ldots, b_{r}\right\}$, and consider an arbitrary basis $\beta_{0}=\left\{b_{1}^{0}, \ldots, b_{r}^{0}\right\}$. Then there exists $i$ such that $\operatorname{Lin}\left\{b_{i}^{0}, \ldots, b_{r}^{0}\right\} \not \subset \operatorname{Lin}\left\{b_{2}, \ldots, b_{r}\right\}$ but $\operatorname{Lin}\left\{b_{i+1}^{0}, \ldots, b_{r}^{0}\right\} \subset \operatorname{Lin}\left\{b_{2}, \ldots, b_{r}\right\}$. For this integer $i$ we have $m_{i}^{\beta_{0}} \geq m_{1}$.

Now we can formulate the main result of this section.

Theorem 6.1: Let the conditions i) and ii) of Theorem 5.2 hold, then

i) The system (4) is null-controllable at the time $T>\bar{m}$;

ii) The system (4) is not controllable at the time $T<m_{1}$.

If the delay is $h$ instead of 1 , then in i) an ii) $\bar{m}$ and $m_{1}$ must be replaced by $\bar{m} h$ and $m_{1} h$ respectively.

\section{Controllability in The General CASE}

In the previous section, we use the assumption that the system (2) is a pure neutral type system $\left(\operatorname{det} A_{-1} \neq 0\right)$. However, this condition is in fact a technical assumption that allows the use of the Riesz basis of eigenspaces of the operator $\mathcal{A}$ in $M_{2}$ and the moment problem approach.

In this section, we show that the conditions i) and ii) are necessary and sufficient for exact controllability for the general neutral systems $\left(A_{-1}\right.$ may be a singular matrix). We obtain also the precise time of controllability. From Theorem 6.1 it is not clear what happens if the time $T$ is such that $m_{1} \leq T \leq \bar{m}$ even if the conditions of controllability are satisfied. In this section, the exact time of controllability is given. In order to do that, we need the classical concept of the controllability indices.

Recall that the first index $n_{1}$ may be defined as the minimal integer $\nu$ such that (see for example [14, Chapter 5])

$$
\operatorname{rank}\left(B, A_{-1} B, \ldots, A_{-1}{ }^{\nu-1} B\right)=n \text {. }
$$

Lemma 7.1: Assume that the pair $\left(\mathcal{A}_{-1}, B\right)$ is controllable. Let $n_{1}$ be the index of controllability of the couple $\left(A_{-1}, B\right)$ and $\bar{m}, m_{1}$ be defined by (14). Then $m_{1} \leq n_{1} \leq$ $\bar{m}$.

It is well known, that in contrast to indices $m_{1}, \bar{m}$, the controllability index $n_{1}$ is invariant under feedback. This means that $n_{1}$ is the same for all couples $\left(A_{-1}+B P, B\right)$, where $P$ is an $r \times n$-matrix. Then one can choose a feedback matrix $P$ and a basis in $\mathbb{C}^{n}$ such that $A_{-1}+B P$ take the following form (see [14, Theorem 5.10 and Corollary 5.3]):

$$
F=\operatorname{diag}\left\{F_{1}, \ldots, F_{r}\right\}
$$

where

$$
F_{i}=\left(\begin{array}{ccccc}
0 & 1 & 0 & \cdots & 0 \\
0 & 0 & 1 & \cdots & 0 \\
\vdots & \vdots & \vdots & \ddots & \vdots \\
0 & 0 & 0 & \cdots & 1 \\
a_{1}^{i} & a_{2}^{i} & a_{3}^{i} & \cdots & a_{n_{i}}^{i}
\end{array}\right)
$$

and $B$ becomes

$$
G=\operatorname{diag}\left\{g_{1}, \ldots, g_{r}\right\},
$$


where $g_{i}=\left(\begin{array}{llll}0 & 0 & \cdots & 1\end{array}\right)^{\mathrm{T}}$, the dimension being $n_{i} \times 1$. It is easy to check that $\bar{m}(F, G)=m_{1}(F, G)=n_{1}$. Moreover, the spectrum of $F$ may be chosen arbitrarily, by means of an appropriate choice of $P$.

Let us now return to the controllability problem for the system (2) (or equivalently (4)). We first give a preliminary result.

Lemma 7.2: The system (2) is exactly null-controllable at the time $T$ if and only if the perturbed system

$$
\dot{z}(t)=\left(A_{-1}+B P\right) \dot{z}(t-1)+L z_{t}+B u,
$$

is exactly null-controllable at the same time $T$, where the operator $L$ is defined as in (3).

Proof: Obviously it is enough to prove one implication only. Assume that the system (2) is controllable at the time $T$. It means that for any function $f(t) \in H^{1}\left(T-1, T ; \mathbb{C}^{n}\right)$ there exists a control $u(t) \in L_{2}\left(0, T ; \mathbb{C}^{n}\right)$ such that the solution of the equation

$$
\dot{z}(t)=A_{-1} \dot{z}(t-1)+L z_{t}+B u(t),
$$

with the initial condition $z(t)=0, t \in[-1,0]$ verifies $z(t)=$ $f(t), t \in[T-1, T]$. Let us rewrite (16) in the form

$$
\dot{z}(t)=\left(A_{-1}+B P\right) \dot{z}(t-1)+L z_{t}+B v(t),
$$

where $v(t)=u(t)-P \dot{z}(t-1), t \in[0, T]$. Since $z(t-1) \in$ $H^{1}\left([0, T] ; \mathbb{C}^{n}\right)$, then $v(t) \in L_{2}\left(0, T ; \mathbb{C}^{n}\right)$. Thus, the control $v(t)$ transfers the state $z(t)=0, t \in[-1,0]$ to the state $z(t)=f(t), t \in[T-1, T]$ by virtue of the perturbed system. This means that it is also controllable at the time $T$.

We have the following result, which concludes our considerations.

Theorem 7.3: Let the neutral type system (2) be in the general form, i.e. without the assumption $\operatorname{det} A_{1} \neq 0$. The conditions i) and ii) of Theorem 5.2 are necessary and sufficient for the exact controllability of the system. Under these conditions, the precise time of controllability is $T=n_{1}$. This means that the system is not controllable for $T \leq n_{1}$ and is controllable for $T>n_{1}$.

If the delay is $h$ instead of 1 , then the exact time of controllability is $n_{1} h$.

\section{CONCLUSION AND PERSPECTIVES}

The main goal of this paper is to demonstrate how the moment problem approach can be used in the controllability problem for delay systems of neutral type. To this end, we chose a quite general model (2) with distributed delays in the function and its derivative, a pointwise neutral term determined by a matrix $A_{-1}$ and the control term by a matrix $B$. Using our approach, we have given a complete analysis of the exact null-controllability for this model. Namely we:

i) showed that the maximal possible set of the states reachable from 0 by the system at some time $T>0$ is the space $H^{1}$,

ii) found the conditions of the parameters of the system under which this set of reachability can be maximally possible (the conditions of exact controllability), iii) proved that, under the above conditions, the system is exactly controllable at the time $T$ if and only if $T>n_{1}$, where $n_{1}$ is the first controllability index of the couple $\left(A_{-1}, B\right)$ (the time of exact controllability).

As a perspective, we consider the extension of our approach to systems with several pointwise neutral terms and to the general case of distributed neutral type delay

$$
K f=\int_{-1}^{0} \mathrm{~d} \mu(\theta) f(\theta), \quad f \in C\left([-1,0], \mathbb{C}^{n}\right),
$$

where $\mu$ is a matrix valued function of bounded variation and continuous at zero. One can prove that, for this class of systems, the generalized Riesz basis property of the model operator $\mathcal{A}$ is preserved. However, the immediate spectral analysis of this operator is more complex. In the case when the delays in the neutral terms are commensurable, the results on exact controllability are expected to be similar to those obtained in the present paper. In the general case, the formulation and the proofs may be much more complicated. This problem is to be considered in our forthcoming works.

\section{REFERENCES}

[1] S. A. Avdonin, S. A. Ivanov, Families of exponentials. The method of moments in controllability problems for distributed parameter systems, Cambridge University Press, Cambridge, 1995.

[2] H. T. Banks, M. Q. Jacobs and C. E. Langenhop, Characterization of the controlled states in $W_{2}^{(1)}$ of linear hereditary systems, SIAM J. Control, 13 (1975), pp. 611-649.

[3] J. A. Burns, T. L. Herdman and H. W. Stech, Linear functionaldifferential equations as semigroups on product spaces, SIAM J. Math. Anal., 14 (1983), pp. 98-116.

[4] K. Ito and T.J. Tarn, A linear quadratic optimal control for neutral systems, Nonlinear Analysis, Theory, Methods \& Applications, 9 (1985), pp. 699-727.

[5] M. Q. Jacobs, C. E. Langenhop, Criteria for function space controllability of linear neutral systems, SIAM J. Control and Optimization, 14 (1976), pp. 1009-1048.

[6] A. Manitius and R. Triggiani, Function space controllability of linear retarded systems: A derivation from abstract operator conditions, SIAM J. on Control and Optimization, 16 (1978), pp. 599-645.

[7] D. A. O'Connor and T. J. Tarn, On the function space controllability of linear neutral systems SIAM J. Control Optimization, 21 (1983), pp. 306-329.

[8] R. Rabah and G. M. Sklyar, The analysis of exact controllability of neutral type systems by the moment problem approach, SIAM J. Control and Optimization, vol. 46 (2007), No6, pp. 2148-2181.

[9] R. Rabah, G. M. Sklyar and A. V. Rezounenko, Generalized Riesz basis property in the analysis of neutral type systems, C. R. Acad. Sci. Paris, Ser.I, 337 (2003), pp. 19-24.

[10] R. Rabah, G. M. Sklyar and A. V. Rezounenko, Stability analysis of neutral type systems in Hilbert space, J. Differential Equations, 214 (2005), pp. 391-428.

[11] R. Rabah, G. M. Sklyar and A. V. Rezounenko, On strong regular stabilizability for linear neutral type systems, J. Differential Equations, 245 (2008), pp. 569593.

[12] Rodas Hernan Rivera, C. E. Langenhop, A sufficient condition for function space controllability of a linear neutral system, SIAM J. Control and Optimization, 16 (1978), pp. 429-435.

[13] D. Ullrich, Divided differences and systems of nonharmonic Fourier series, Proc. of American Math. Society, 80 (1980), pp. 47-57.

[14] W. M. Wonham, Linear multivariable control: A geometric approach, Springer, New York, 1985.

[15] Y. Yamamoto, Reachability of a class of infinite-dimensional systems: an external approach with applications to general neutral systems, SIAM J. Control and Optimization, 27 (1989), pp. 217-234.

[16] R. Y. Young, An introduction to nonharmonic analysis, Academic Press, New York, 1980. 